\title{
The role of pain-mediating sensory nerves in histamine-induced inflammation of nasal mucosa*
}

\author{
Henrik Waldenström ${ }^{1,2}$, Magnus Backheden ${ }^{3}$, Jan Erik Juto ${ }^{1,2}$ \\ Department of Otorhinolaryngology, Karolinska University Hospital/Huddinge, Stockholm, Sweden \\ Department of Clinical Sciences, Intervention and Technology, Karolinska Institutet, Stockholm, Sweden \\ Department of Learning, Informatics, Management \& Ethics, Karolinska Institutet, Stockholm, Sweden
}

SUMMARY

\begin{abstract}
Topical application of histamine on the nasal mucosa causes an inflammatory reaction with increased mucosal swelling and perfusion. In the nasal mucosa histamine receptors are found in the vascular epithelium and at free sensory nerve endings. The aim of this randomized double-blind placebo controlled study was to investigate if this inflammatory reaction to locally administered histamine was dependent upon the stimuli of pain-mediating sensory nerves, or if it mainly was the result of direct stimuli of the vascular epithelium. Eighteen healthy non-allergic subjects were treated with sprays of lidocaine or saline (placebo) in the nose, followed by challenge with histamine. Using a cross-over design the participants later returned and were allocated to the opposite treatment followed by histamine. Nasal congestion, and concentration, velocity and perfusion of erythrocytes were measured with rhinostereometry and laser Doppler flowmetry. Symptoms of blockage, discharge and itching were scored. When comparing the lidocaine group to the placebo group, we found no significant differences in the reaction to histamine concerning any of the measured parameters or subjective symptoms. In conclusion, our results suggest that the stimuli of pain-mediating sensory nerves do not significantly contribute to the inflammatory effect of histamine on human nasal mucosa.
\end{abstract}

Key words: sensory nerves, neurogenic inflammation, nasal mucosa, histamine, lidocaine, rhinostereometry, laser-Doppler flowmetry

\section{INTRODUCTION}

Idiopathic rhinitis (IR), formerly also called vasomotor rhinitis, is a diagnosis of exclusion and is given to patients suffering from perennial nasal congestion, rhinorrhea and or sneezing with no identifiable aetiology. One hypothesis is that the underlying pathophysiology of IR is a hyperactive sensory neural system. Stimulation of these nerves, mainly unmyelinated C-fibers, causes a local release of neuropeptides, which might contribute to the symptoms of IR ${ }^{(1-4)}$. These sensory Cfibers are sensitive to capsaicin ${ }^{(5,6)}$ and repeated application of capsaicin leads to desensitization and degeneration of the fibres ${ }^{(7,8)}$. Several studies have been published showing a therapeutic effect in IR patients for repeated topical application of capsaicin ${ }^{(9-14)}$.

Apart from capsaicin, stimulation of the sensory C-fibers can be induced by a number of inhaled irritants and by inflammatory mediators like histamine ${ }^{(15-17)}$. In the nasal mucosa histamine receptors are found in the vascular epithelium and at free nerve endings ${ }^{(18)}$. Topical application of histamine on the nasal mucosa causes an inflammatory reaction with increased mucosal swelling and perfusion ${ }^{(19,20)}$. The aim of this study was to investigate if this inflammatory reaction to locally administered histamine was dependent upon the stimuli of pain-mediating sensory nerves, or if it mainly was the result of direct stimuli of the vascular epithelium.

\section{METHODS}

Patients

The study was conducted as a randomized double blinded controlled trial using cross-over design. Participants to the study were recruited via advertisements (notes) in a large university hospital and two university campuses in Stockholm, Sweden. The inclusion criteria were: age 20-40 years, good health, no medication or contraceptive pills, not pregnant, no allergy, no chronic rhinitis of any kind, and no smoking or snuffing. The exclusion criteria were pronounced septal deviation and poor dental status, which both would make good readings with the rhinostereometer impossible. Each person was tested for any non-symptomatic allergy against birch pollens, timothy pol- 
lens, mugwort pollens, canine, cat, horse, mold and mite with skin prick test Soluprick (ALK, Denmark), and excluded if tested positive. In total, 24 participants were recruited to the study. Two withdrew and 1 became pregnant before randomization. After randomization, 2 were excluded because of administrative errors and 1 because of initiation of medication.

\section{Rhinostereometry}

For registrations of the reactions of the nasal mucosa we used a rhinostereometer (Rhinomed, Sweden). Rhinostereometry (RSM) is a direct optical method where the subject is secured to a frame by means of an individually adapted dental splint. It enables registrations of changes in swelling of the mucosa with an accuracy of $0,2 \mathrm{~mm}$ at different measurements over time. The details of the measuring technique have been described elsewhere (21).

\section{Laser Doppler flowmetry}

Laser Doppler flowmetry (LDF) was performed using a Periflux 4001 (Perimed, Sweden). The laser Doppler penetrates the superficial vascular bed of the mucosa and enables calculations of the concentration (which reflects the level of oedema), velocity and perfusion of erythrocytes. The wavelength of the laser beam was $780 \mathrm{~nm}$. A specially designed probe was used with an outer diameter of $1,6 \mathrm{~mm}$ and a fibre separation of 0,5 $\mathrm{mm}$. The surface of the probe end was angled $15^{\circ}$ from the line of sight in order to keep this surface parallel to the mucosa. A rhinostereometer equipped with a micromanipulator (Rhinomed) was used to combine the two methods. Thus it was possible to position and, if the mucosal swelling altered, continuously adjust the laser probe end to the desired distance to the mucosal surface to be studied. The probe is placed in the plane of focus and can then be adjusted in the dimensions up-down and side to side with an accuracy of $0,1 \mathrm{~mm}$. Should the surface diverge from the vertical plane, the probe can be turned around its axis in order to maintain parallelism. In this way it was possible to keep the measuring distance stable dur-

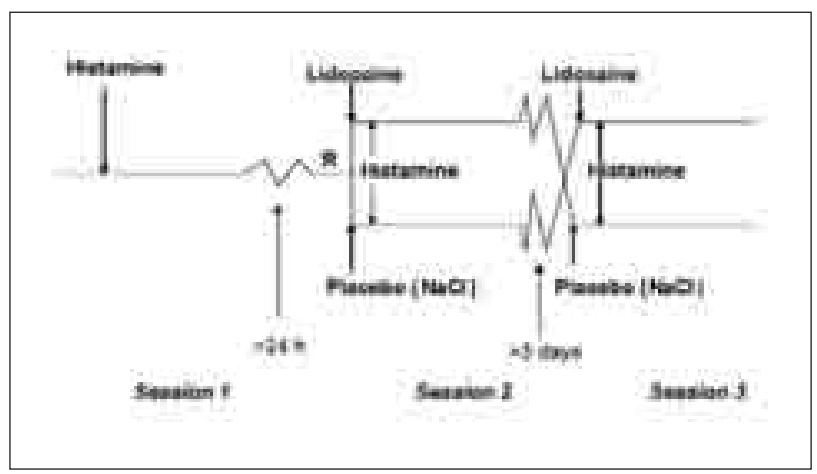

Figure 1. Study design. After each administration of histamine, lidocaine and placebo, nasal symptoms were scored and the degree of congestion according to RSM and the LDF parameters perfusion, concentration and velocity were measured repeatedly. ( $\mathrm{R}=$ randomization). ing the registration of flowmetry values. The signal was fed into an IBM compatible computer using the Perisoft software program.

\section{Substances}

Three substances were used in our study: lidocaine, histamine and placebo. Forty mg lidocainehydrochlorid was diluted with $(9 \mathrm{mg} / \mathrm{ml})$ isotonic saline to a concentration of $40 \mathrm{mg} / \mathrm{ml}$. Four $\mathrm{mg}$ histaminedihydrochloride was diluted with sterile water to a concentration of $2 \mathrm{mg} / \mathrm{ml}$. Isotonic saline $(9 \mathrm{mg} / \mathrm{ml})$ was used as placebo. None of the solutions contained preservatives.

\section{Trial}

The participants and the person administering the intervention (HW) were blinded to the group assignment. The participants chose an opaque envelope from a box including 24 prepared envelopes with 12 paper strips typed with an A (control group) and 12 with a $\mathrm{B}$ (experimental group).

The trial consisted of three different sessions. If a participant caught a cold, a session was postponed two weeks or more after complete relief of any nasal symptoms. All subjects were allowed to acclimatize for at least $30 \mathrm{~min}$ before the start of each session, and a session did not start until three consecutive measures of mucosal congestion with two minutes in between, varied with less than $0,4 \mathrm{~mm}$ according to RSM.

One week or more after the skin prick test the participants were called to the first session (Figure 1, session 1). The subjects were then challenged with $0,14 \mathrm{ml}$ histaminedihydrochlorid $(2 \mathrm{mg} / \mathrm{ml})$ that was dripped onto the surface of the inferior turbinate in the right nostril, at the exact portion of the mucosa being studied. After administration the degree of congestion according to RSM and the LDF parameters perfusion, concentration and velocity were measured. The measurements were repeated $2,5,10,15$, and $20 \mathrm{~min}$ after the challenge. The reason for this first session was to make the participants familiar with the setting and procedure of the experiment. This is important since emotional stress affects circulation and congestions of the nasal mucosa. The first session was also used as a wash-out before randomization.

At the second session, which took place at least one day after the first session, the participants were randomized into an experimental and control group, respectively. The experimental group was given lidocainehydrochlorid $(40 \mathrm{mg} / \mathrm{ml})$, in order to block the pain-mediating sensory nerves, and the control group received placebo containing saline $(9 \mathrm{mg} / \mathrm{ml}$ ) (Figure 1, session 2). The lidocaine and saline solutions had identical packing only marked with an A or a B. The solutions were administered in a nasal spray in the right nostril. Two sprays were given and each spray contained $4 \mathrm{mg}$ lidocainehydrochlorid in the experimental group and $0,9 \mathrm{mg}$ saline in the placebo group. Two and 5 min after administration, the degree of con- 


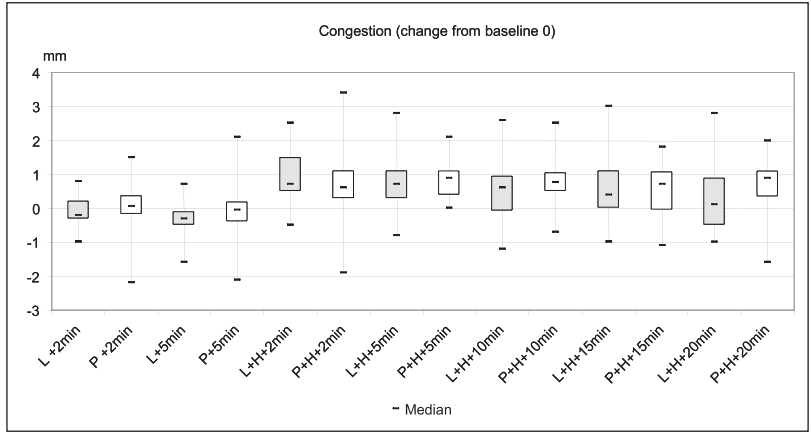

Figure 2. Changes in congestion following local application of lidocaine $(\mathrm{L})$ or placebo $(\mathrm{P})$ followed by histamine challenge $(\mathrm{H})$. A significant trend of increased swell after histamine challenge can be seen in both groups $(\mathrm{p}<0,0001)$, but there is no significant difference in the reaction to histamine in the lidocaine group compared to placebo. (The boxes boundaries indicate the upper and lower quartile, and the whiskers indicate the largest and smallest observation).

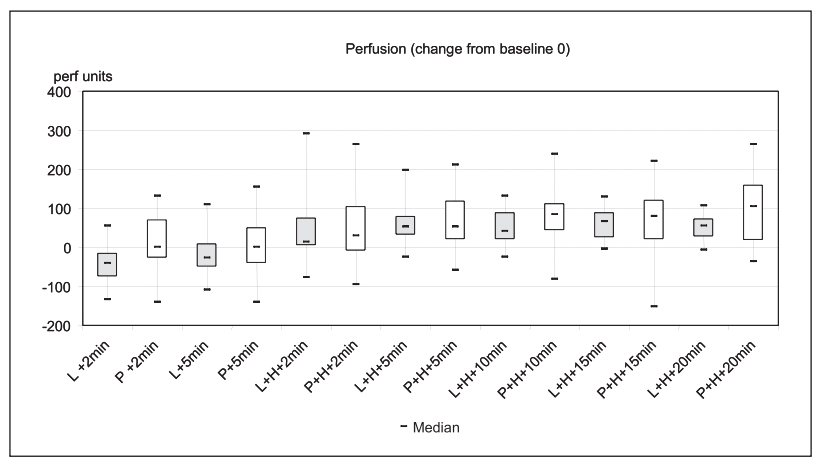

Figure 3. Changes in perfusion following local application of placebo $(\mathrm{P})$ or lidocaine $(\mathrm{L})$ followed by histamine challenge $(\mathrm{H})$. A significant trend of increased perfusion after histamine challenge can be seen in both groups $(p<0,0001)$, but there is no significant difference in the reaction to histamine in the lidocaine group compared to placebo. (The boxes boundaries indicate the upper and lower quartile, and the whiskers indicate the largest and smallest observation).

gestion according to RSM and the LDF parameters, perfusion, concentration and velocity were measured. The subjects were then challenged with histamine in the same manner as in session 1 . The measurements were repeated $2,5,10,15$, and 20 min after the challenge. After a period of at least 3 days the participants returned and were allocated to the opposite treatment (Figure 1, session 3).

At every session, symptoms of nasal blockage, discharge and itching were scored on an ordinal scale (1-4) at each time of measurement.

\section{Statistical analysis}

For statistical calculations of RSM and LDF readings we used a mixed effects model for longitudinal data (PROCEDURE MIXED, SAS). The difference between lidocaine and placebo and the general trend over time as well as the interaction between trend over time and treatment were estimated and evaluated in the statistical analyses. In the mixed effects models both treatment and time points were analysed as within effects, due to the crossover design. The covariance matrix, direct product CS was used in the analyses. The changes in the subjective scores of nasal blockage, discharge and itching were analysed, in dichotomised versions, with the McNemar test. A value for $\mathrm{p}<0,05$ was considered statistically significant.

\section{RESULTS}

Challenge with histamine without pre-treatment with placebo or lidocaine (session 1) resulted in an increased congestion and perfusion. Since it was not the aim of the study, these results were not statistically analyzed.

A prompt inflammatory reaction after histamine provocation was seen in the placebo and lidocaine groups (session 2 and 3), with significant trends of increased swell ( $p<0,0001)$, decreased concentration ( $\mathrm{p}<0,008)$, increased velocity $(\mathrm{p}<0,0001)$ and perfusion $(\mathrm{p}<0,0001)$ in both groups.

We compared every parameter in the placebo group to the corresponding parameters in the lidocaine group by analyzing potential differences in trends stretching over the whole period from 2 to 20 minutes after administration of histamine, and we also looked for differences at each time of reading. We found no significant differences concerning any of the measured parameters.

At the 2 min reading, before challenge with histamine, there was a tendency for decreased velocity $(\mathrm{p}=0,06)$ and perfusion $(\mathrm{p}=0,08)$ in the lidocainegroup compared to placebo. This tendency was much weaker at $5 \mathrm{~min}$ and could not be seen at the subsequent readings. This finding might be due to a possible vasoconstrictive action of lidocaine, which has been reported from clinical findings ${ }^{(22)}$.

The reactions to histamine tended to be more homogenous following lidocaine treatment compared to placebo, with less variance in the lidocaine group for all parameters. Although none of these differences, when analyzed separately, were significant.

The participants scored a significantly higher degree of nasal blockage 5 minutes after administration of lidocaine compared to placebo $(\mathrm{p}<0,05)$ (before challenge with histamine). Two minutes after administration of histamine, the placebo group reacted with a significant increase of subjective nasal blockage $(\mathrm{p}<0,001)$, whereas the increase of blockage in the lidocaine group was on the verge of being significant $(p=0.065)$. At no time after challenge with histamine were there any differences between the groups in their scoring of blockage.

There was a significant increase of subjective itching in the 


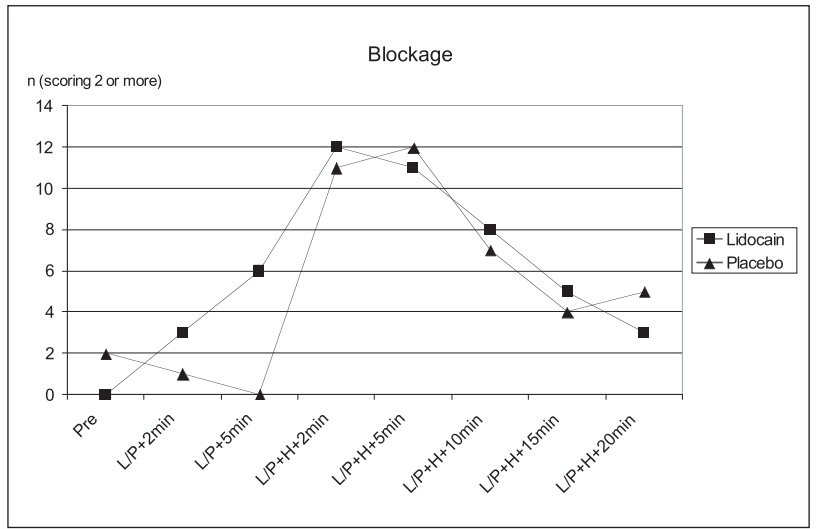

Figure 4. Numbers of participants scoring 2 or more, when asked to score their subjective sense of nasal blockage on an ordinal scale 1-4. Scorings were made before and after local application of lidocaine (L) or placebo $(\mathrm{P})$ followed by histamine challenge $(\mathrm{H})$.

lidocaine group 2 minutes after the administration of histamine $(\mathrm{p}<0.01)$. The increase of itching in the placebo group at the same time was not significant $(\mathrm{p}=0,13)$. When comparing the two groups, no significant differences could be seen at any time.

There was a significant increase of subjective discharge in both groups 2 minutes after the administration of histamine (lidocaine: $\mathrm{p}<0.01$, placebo: $\mathrm{p}<0.001)$ No differences between the groups could be seen at any time.

\section{DISCUSSION}

We found no significant differences between the histamine reactions following lidocaine treatment compared to placebo. Thus this study implies that the pain-mediating sensory nerves do not significantly contribute to the inflammatory effect of histamine on human nasal mucosa.

Our study had some limitations. Six participants, who were all measured in the beginning of the study, had lower absolute levels of concentration overall. We believe that the most plausible explanation is a re-calibration of the laser Doppler that had to be done following a repair of the laser probe. Since our analysis for every individual parameter is based not on absolute values but on differences from the measured baseline value at each session, differences in absolute numbers would be no problem as long as the sensitivity of the apparatus was consistent. To address this question we plotted the differences for each measured value to the preceding one (e.g. value at 5 min after histamine - value at $2 \mathrm{~min}$ after histamine), for all parameters at all times of measurement, and compared these 6 participants to the others by descriptive box-plots. We could see no sign of systematic differences between the groups.

Did we not use enough lidocaine? We believe that we used enough lidocaine to at least partially block the pain-mediating

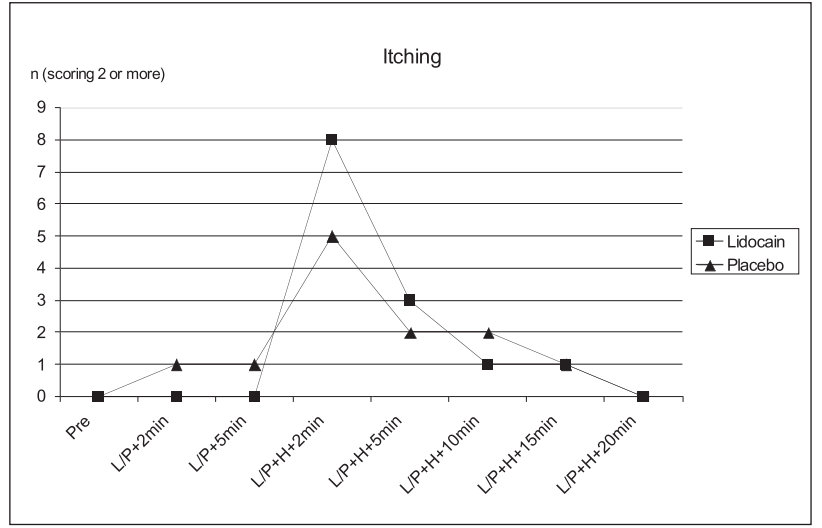

Figure 5. Numbers of participants scoring 2 or more, when asked to score their subjective sense of nasal itching on an ordinal scale 1-4. Scorings were made before and after local application of lidocaine (L) or placebo $(\mathrm{P})$ followed by histamine challenge $(\mathrm{H})$.

sensory nerves, based on clinical experience when lidocaine is used in nasal spray for anaesthesia. Further, the lidocaine dose given affected the sensory nerves with increased scores of blockage before challenge with histamine, compared to placebo. The question can also be raised if we used too much histamine so the stimulus of histamine receptors in the vascular bed overshadowed any differences between the groups. The dose of histamine was based on earlier studies where we have seen that this dose causes an inflammatory reaction of reasonable proportions ${ }^{(19,20)}$. Each subject reacted with an increased swell of the nasal mucosa following challenge with this dose of histamine.

The role of the nervous system in histamine-induced inflammation of the nasal mucosa has been investigated in earlier studies, to some extent with conflicting results. Okuda ${ }^{(23)}$ found that the sneezing and the secretor responses induced by histamine were abrogated by topical anaesthesia with cocaine, supporting the role of the nervous system. Vidian neurectomy has been shown to diminish the secretion followed by stimulation with histamine, without affecting the increase of mucosal swelling, suggesting that the secretion would be the result of a central reflex ending in parasympathetic stimulation of glands ${ }^{(24)}$. On the other hand, Raphael ${ }^{(25)}$ found that topical pre-treatment with chlorpheniramine (an H-1 antihistamine) completely abrogated the ipsilateral nasal secretor response to histamine, whereas nasal pre-treatment with atropine (a muscarinic antagonist) had no significant effect. Raphael also found that histamine produced a smaller contralateral protein secretion which contained disproportionately elevated concentrations of the glandular protein SIgA, and they concluded that histamine seems to stimulate secretion by both a direct action on $\mathrm{H}-1$ receptors and by an indirect reflex mechanism that stimulates glandular secretion. This view was supported by Baroody ${ }^{(26)}$. They found that histamine induced a dose-dependent increase in secretion on the challenged side, and it also induced a neur- 
al reflex with secretion on the contralateral side. Pre-treatment with atropine resulted in inhibition of the contralateral secretory response and partial inhibition of the ipsilateral response. Terfenadine (antihistamine) pre-treatment resulted in complete inhibition of both the ipsilateral and contralateral response. In summary, the study of Baroody implies that histamine acts by both direct and neurogenic stimulation. Most of these earlier studies have quantified the inflammatory response in terms of nasal secretion and sometimes scoring of symptoms. A study like ours, with repeated readings of mucosal swelling, perfusion and oedema at the exact same point of the nasal mucosa, has never been done before in this field of research. When speaking of neurogenic stimulation, one must remember that this study is limited by its focus on pain-mediating sensory nerves, upon which lidocaine has its main inhibiting effect. Perhaps stimulation of sensory nerves mediating other modalities like temperature or pressure constitutes a larger contribution to the inflammatory effect of histamine. Studies designed to address this question would be of interest in the future.

\section{REFERENCES}

1. Baraniuk JN, Kaliner MA. Neuropeptides and nasal secretion. J Allergy Clin Immunol. 1990; 86: 620-627.

2. Fang SY, Shen CL. Neuropeptide innervation and neuroendocrine cells in allergic rhinitis and chronic hypertrophic rhinitis. Clin Exp Allergy. 1998; 28: 228-232.

3. Lacroix JS, Kurt AM, Pochon N, Bretton C, Lundberg JM, Deshusses J. Neutral endopeptidase activity and concentration of sensory neuropeptide in the human nasal mucosa. Eur Arch Otorhinolaryngol. 1995; 252; 465-466.

4. Lacroix JS, Landis BN. Neurogenic inflammation of the upper airway mucosa. Rhinology. 2008; 46: 163-165.

5. Jancsó N, Jancsó-Gábor A, Szolcsányi, J. Direct evidence for neurogenic inflammation and its prevention by denervation and by pre-treatment with capsaicin. Br J Pharmacol Chemother. 1967; 31: 138-151.

6. Holzer P. Local effector functions of capsaicin-sensitive sensory nerve endings: involvement of tachykinins, calcitonin gene-related peptide and other neuropeptides. Neuroscience 1988; 24: 739-768.

7. Holzer P. Capsaicin: cellular targets, mechanisms of action, and selectivity for thinensory neurons. Pharmacol Rev. 1991; 43: 143201.

8. Kitajiri M, Kubo N, Ikeda H, Sato K, Kumazawa T. Effects of topical capsaicin on autonomic nerves in experimentally-induced nasal hypersensitivity. An immunocytochemical study. Acta Otolaryngol Suppl (Stockh). 1993; 500: 88-91.

9. Lacroix JS, Buvelot JM, Polla BS, Lundberg JM. Improvement of symptoms of non-allergic chronic rhinitis by local treatment with capsaicin. Clin Exp Allergy. 1991; 21: 595-600.
10. Blom HM, Van Rijswijk JB, Garrelds IM, Mulder PG, Timmermans T, Gerth van Wijk R. Intranasal capsaicin is efficacious in non-allergic, non-infectious perennial rhinitis. A placebocontrolled study. Clin Exp Allergy. 1997; 27: 796-780.

11. Wolf G, Anderhuber W, Hauser-Kronberger C, Saria A. Treatment of nonspecific hyper-reflectory rhinopathy (vasomotor rhinitis) with capsaicin. Laryngorhinootologie. 1995; 74: 289-293.

12. Riechelmann H, Davris S, Bader D. Treatment of perennial nonallergic rhinopathy with capsaicin. HNO. 1993; 41: 475-479.

13. Marabini S, Ciabatti PG, Polli G, Fusco BM, Geppetti P. Beneficial effects of intranasal applications of capsaicin in patients with vasomotor rhinitis. Eur Arch Otorhinolaryngol. 1991; 248: 191-194.

14. Van Rijswijk JB, Boeke EL, Keizer JM, Mulder PG, Blom HM, Fokkens WJ. Intranasal capsaicin reduces nasal hyperreactivity in idiopathic rhinitis: a double-blind randomized application regimen study. Allergy. 2003; 58: 754-761.

15. Svensson C, Andersson M, Greiff L, Persson CG. Nasal mucosal endorgan hyperresponsiveness. Am J Rhinol. 1998; 12: 37-43.

16. Änggård A. Basic mechanisms in autonomic nervous responses in specific and nonspecific nasal hyperreactivity. Acta Otolaryngol (Stockh). 1993; 113: 394-396.

17. Sanico AM, Atsuta S, Proud D, Togias A. Dose-dependent effects of capsaicin nasal challenge: in vivo evidence of human airway neurogenic inflammation. J Allergy Clin Immunol. 1997; 100: 632641.

18. Mygind N. Essential Allergy. Oxford: Blackwell Science, 1996.

19. Hallén H, Juto JE. Nasal mucosa reaction. A model for mucosal reaction during challenge. Rhinology 1992; 30; 129-133.

20. Grudemo H, Juto JE. Rhinostereometry and laser Doppler flowmetry in human nasal mucosa. Changes in congestion and microcirculation during intranasal histamine challenge. ORL. 1997; 59: 50-56.

21. Juto JE, Lundberg C. An optical method for determining changes in mucosal congestion in the nose in man. Acta Otolaryngol. 1982; 94: $149-156$

22. Merrill DG, Brodsky JB, Hentz RV. Vascular insufficiency following axillary block of the brachial plexus. Anesth Analg. 1981; 60: 162-164.

23. Okuda M, Ohtsuka H, Sakaguchi K, Watase T. Nasal histamine sensitivity in allergic rhinitis. Ann Allergy. 1983; 51: 51-55.

24. Konno A, Togawa K. Role of the vidian nerve in nasal allergy. Ann Otol Rhinol Laryngol. 1979; 88: 258-266.

25. Raphael GD, Meredith SD, Baraniuk JN, Druce HM, Banks SM, Kaliner MA. The pathophysiology of rhinitis. II. Assessment of the sources of protein in histamine-induced nasal secretions. Am Rev Respir Dis. 1989; 139: 791-800.

26. Baroody FM, Wagenmann M, Naclerio RM. Comparison of the secretory response of the nasal mucosa to methacholine and histamine. J Appl Physiol. 1993; 74: 2661-2671.

Henrik Waldenström MD

Department of Otorhinolaryngology

Karolinska University Hospital/Huddinge

S-141 86 Stockholm

Sweden 